\title{
The Weighted New Weibull Pareto Distribution: Some Characteristics and Applications
}

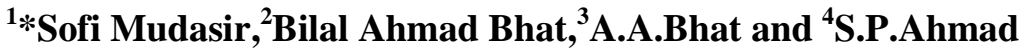 \\ ${ }^{1,2,3,4}$ Department of Statistics, University of Kashmir, Srinagar, India \\ *Corresponding email: sofimudasir3806@gmail.com
}

Available online at: www.isroset.org

Received:06/Oct /2018, Accepted:15/Oct/2018, Online: 31/Oct/2018

\begin{abstract}
In this paper, a weighted version of New Weibull Pareto (NWP) distribution known as weighted new Weibull Pareto (WNWP) distribution is obtained. Some structural properties of the new model are studied. Applications are provided using two real life data sets. It is shown that our new model performs better as compared to other models.
\end{abstract}

Keywords: Statistical distributions, reliability measures, Shannon's entropy and real data sets.

\section{INTRODUCTION}

In real life, there exist some situations when for an investigator it is not possible to select a sample with equal probability. In such situations, sampling frames are not properly defined and recorded observations are biased and do not follow the original distribution. Modeling of these observations gives birth to the theory of weighted distributions which was given by Fisher [1] and then studied by Rao [2].There are many authors who have presented important results on weighted distributions among them are Jain et al. [3] introduced the weighted version of gamma distribution, Abd El-Moonsef and Ghoneim [4] studied the weighted version of Kumaraswamy distribution, Fatima and Ahmad [5] introduced the weighted form of inverse Rayleigh distribution and study its various properties, Sofi Mudasir and Ahmad [6] proposed the weighted version of Nakagami distribution and finds its application to real life, Sofi Mudasir and Ahmad [7] estimate the scale parameter of weighted Erlang distribution through classical and Bayesian methods of estimation, Jan et al. [8] studied the weighted Ailamujia distribution and find its applications to life time data.

If $V \geq 0$ is a random variable with density function $f(v)$ and $w(v, \theta) \geq 0$ is a weight function, then the weighted random variable $V_{W}$ has the density function given by

$$
f_{w}(v)=Z w(v, \theta) f(v)
$$

Where $\mathrm{Z}$ is the normalizing constant.

When $w(v, \theta)=v^{\theta}, \theta>0$, then the distribution is called the weighted distribution of order $\theta$.

The probability density function of NWP distribution given by Nasiru and Luguterah [9] is given as

$$
f(v)=\frac{\beta \eta}{\alpha}\left(\frac{v}{\alpha}\right)^{\beta-1} \exp \left(-\eta\left(\frac{v}{\alpha}\right)^{\beta}\right), v>0 ; \alpha, \beta, \eta>0 .
$$

By using eq. (1.2) and $w(v)=v^{\theta}$ in eq. (1.1), we get the required pdf of WNWP distribution and is given by

$$
f_{w}(v)=\frac{\beta \eta^{\frac{\theta}{\beta}+1}}{\alpha^{\beta+\theta} \Gamma\left(\frac{\theta}{\beta}+1\right)} v^{\beta+\theta-1} \exp \left(-\eta\left(\frac{v}{\alpha}\right)^{\beta}\right), v>0 ; \alpha, \beta, \theta, \eta>0 .
$$




\section{SUB-MODELS}

(i) If in eq. (1.3) $\alpha=1, \theta=0$, we get the Weibull distribution with pdf given as

$$
f(v)=\beta \eta v^{\beta-1} \exp \left(-\eta v^{\beta}\right) .
$$

(ii) If $\theta=0$, in eq.(1.3) we get the basic model given in eq. (1.2).

(iii) When in eq.(1.3), $\theta=1$, the WNWP distribution reduces to length-biased new Weibull Pareto (LNWP) distribution with pdf given as

$$
f_{l}(v)=\frac{\beta \eta^{\frac{1}{\beta}+1}}{\alpha^{\beta+1} \Gamma\left(\frac{1}{\beta}+1\right)} v^{\beta} \exp \left(-\eta\left(\frac{v}{\alpha}\right)^{\beta}\right) .
$$

(iv) When in eq. (1.3), $\theta=2$, the WNWP distribution reduces to area-biased new Weibull Pareto (ANWP) distribution with pdf given as

$$
f_{a}(v)=\frac{\beta \eta^{\frac{2}{\beta}+1}}{\alpha^{\beta+2} \Gamma\left(\frac{2}{\beta}+1\right)} v^{\beta+1} \exp \left(-\eta\left(\frac{v}{\alpha}\right)^{\beta}\right) .
$$

\section{FUNCTIONS RELATED TO WNWP DISTRIBUTION}

Proposition 1. Let V be a r.v. with pdf given in (1.3). The associated cumulative distribution function (cdf) is given by:

$$
F(v)=\frac{1}{\Gamma\left(\frac{\theta}{\beta}+1\right)} \gamma\left(\frac{\theta}{\beta}+1, \eta\left(\frac{v}{\alpha}\right)^{\beta}\right) .
$$

Proof. Using the definition of cdf, we find that

$$
F(v)=\frac{\beta \eta^{\frac{\theta}{\beta}+1}}{\alpha^{\beta+\theta} \Gamma\left(\frac{\theta}{\beta}+1\right)} \int_{0}^{v} v^{\beta+\theta-1} \exp \left(-\eta\left(\frac{v}{\alpha}\right)^{\beta}\right) d v .
$$

By substituting $y=\eta\left(\frac{v}{\alpha}\right)^{\beta}$, we get

$$
F(v)=\frac{1}{\Gamma\left(\frac{\theta}{\beta}+1\right)} \gamma\left(\frac{\theta}{\beta}+1, \eta\left(\frac{v}{\alpha}\right)^{\beta}\right) \text {. }
$$

This completes the proof. 

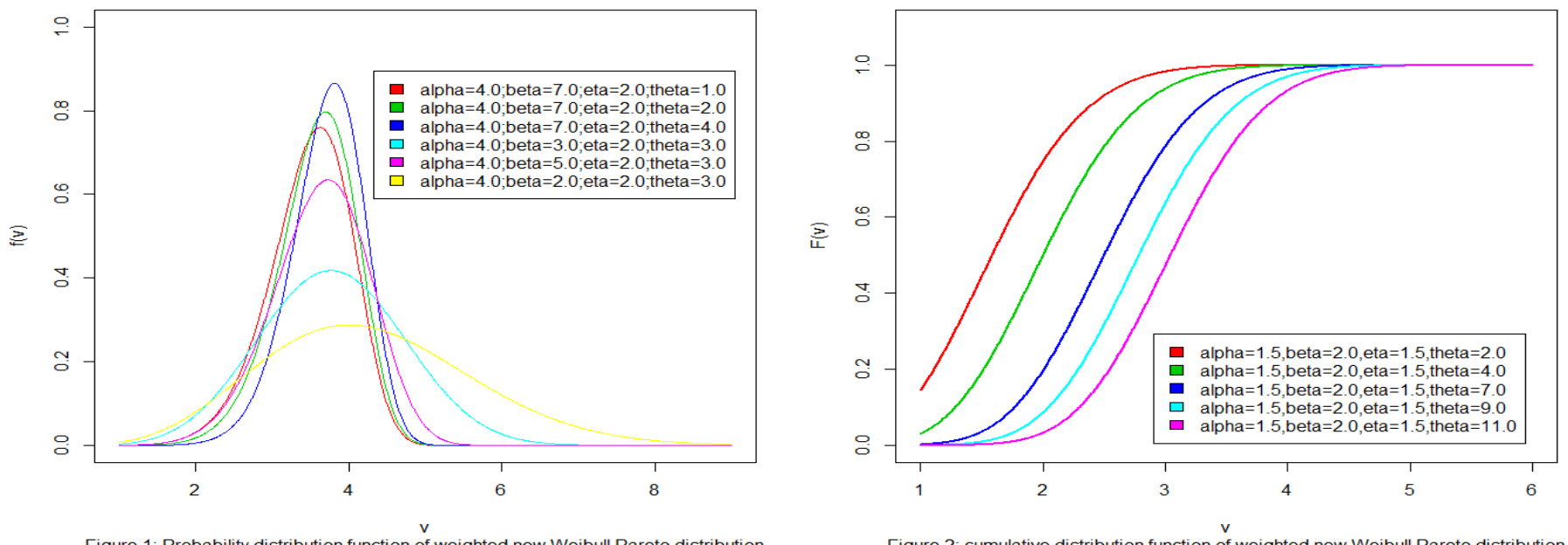

The survival and hazard rate functions follow immediately:

$$
\begin{aligned}
& S(v)=1-\frac{1}{\Gamma\left(\frac{\theta}{\beta}+1\right)} \gamma\left(\frac{\theta}{\beta}+1, \eta\left(\frac{v}{\alpha}\right)^{\beta}\right) . \\
& h(v)=\frac{\beta \eta^{\frac{\theta}{\beta}+1} v^{\beta+\theta-1} \exp \left(-\eta\left(\frac{v}{\alpha}\right)^{\beta}\right)}{\alpha^{\beta+\theta}\left(\Gamma\left(\frac{\theta}{\beta}+1\right)-\gamma\left(\frac{\theta}{\beta}+1, \eta\left(\frac{v}{\alpha}\right)^{\beta}\right)\right)} .
\end{aligned}
$$

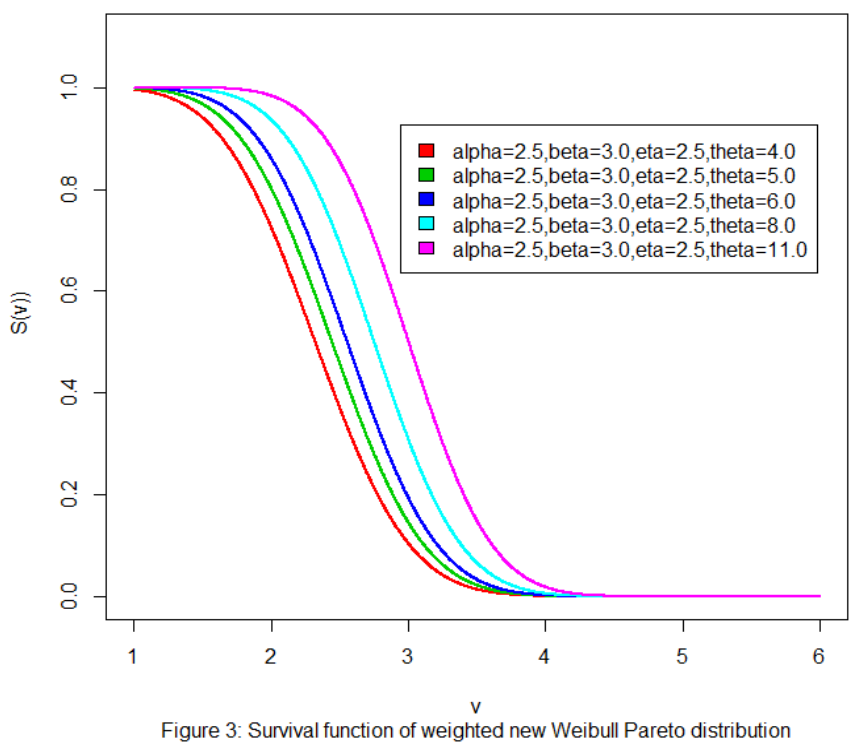

Figure 3: Survival function of weighted new Weibull Pareto distribution

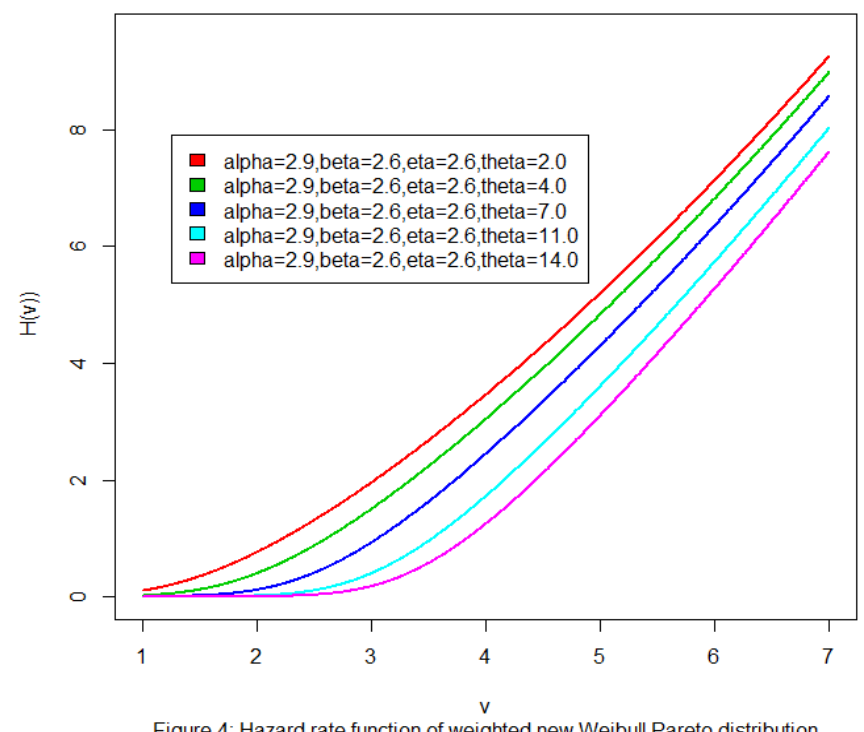

Figure 4: Hazard rate function of weighted new Weibull Pareto distribution 


\section{ASYMPTOTIC BEHAVIOR}

Here it can be checked by finding out the value of $\lim _{v \rightarrow \infty} f_{w}(v)$ and $\lim _{v \rightarrow 0} f_{w}(v)$ as follows

$$
\begin{aligned}
\lim _{v \rightarrow \infty} f_{w}(v) & =\lim _{v \rightarrow \infty} \frac{\beta \eta^{\frac{\theta}{\beta}+1}}{\alpha^{\beta+\theta} \Gamma\left(\frac{\theta}{\beta}+1\right)} v^{\beta+\theta-1} \exp \left(-\eta\left(\frac{v}{\alpha}\right)^{\beta}\right) \\
= & \frac{\beta \eta^{\frac{\theta}{\beta}+1}}{\alpha^{\beta+\theta} \Gamma\left(\frac{\theta}{\beta}+1\right)^{\beta \rightarrow \infty}} \lim ^{\beta+\theta-1} \lim _{v \rightarrow \infty} \exp \left(-\eta\left(\frac{v}{\alpha}\right)^{\beta}\right)=0 .
\end{aligned}
$$

And $\lim _{v \rightarrow 0} f_{w}(v)=\lim _{v \rightarrow 0} \frac{\beta \eta^{\frac{\theta}{\beta}+1}}{\alpha^{\beta+\theta} \Gamma\left(\frac{\theta}{\beta}+1\right)} v^{\beta+\theta-1} \exp \left(-\eta\left(\frac{v}{\alpha}\right)^{\beta}\right)$

$$
=\frac{\beta \eta^{\frac{\theta}{\beta}+1}}{\alpha^{\beta+\theta} \Gamma\left(\frac{\theta}{\beta}+1\right)} \lim _{v \rightarrow 0} v^{\beta+\theta-1} \lim _{v \rightarrow 0} \exp \left(-\eta\left(\frac{v}{\alpha}\right)^{\beta}\right)=0 .
$$

Whenever, $v \rightarrow \infty$ and $v \rightarrow 0$, then the pdf also tends to zero. Hence the WNWP distribution has mode.

\section{STATISTICAL PROPERTIES}

This section deals with the statistical properties of WNWP distribution.

5.1. Mode of WNWP distribution

The mode of the WNWP distribution can be found by solving the equation $\frac{\partial}{\partial v}\left(\log \left(f_{w}(v)\right)\right)=0$. Therefore, the mode at $v=v_{0}$ is given by

\subsection{Moments}

$$
v_{0}=\alpha\left(\frac{\beta+\theta-1}{\eta \beta}\right)^{\frac{1}{\beta}} .
$$

Proposition 2. Let $\mathrm{V}$ be a r.v. with pdf given by (3). Then the $\mathrm{r}^{\text {th }}$ non-central moment is given by

$$
\mu_{r}^{\prime}=\frac{\alpha^{r} \Gamma\left(\frac{\theta+r}{\beta}+1\right)}{\eta^{\frac{r}{\beta}} \Gamma\left(\frac{\theta}{\beta}+1\right)}=\frac{\alpha^{r} \rho_{\theta+r}}{\eta^{\frac{r}{\beta}} \rho_{\theta}} .
$$

Where $\rho_{\theta+s}=\Gamma\left(\frac{\theta+s}{\beta}+1\right)$.

Proof. According to (3) 


$$
\mu_{r}^{\prime}=\frac{\beta \eta^{\frac{\theta}{\beta}+1}}{\alpha^{\beta+\theta} \Gamma\left(\frac{\theta}{\beta}+1\right)} \int_{0}^{\infty} v^{\beta+\theta+r-1} \exp \left(-\eta\left(\frac{v}{\alpha}\right)^{\beta}\right) d v
$$

After the simplification of the above integral, we get

$$
\mu_{r}^{\prime}=\frac{\alpha^{r} \Gamma\left(\frac{\theta+r}{\beta}+1\right)}{\eta^{\frac{r}{\beta}} \Gamma\left(\frac{\theta}{\beta}+1\right)}=\frac{\alpha^{r} \rho_{\theta+r}}{\eta^{\frac{r}{\beta}} \rho_{\theta}} .
$$

This ends the proof.

By using eq. (5.2.1), the mean and variance of WNWP distribution are given by

$$
\text { Mean }=\frac{\alpha \rho_{\theta+1}}{\eta^{\frac{1}{\beta}} \rho_{\theta}} \text { and variance }=\left(\frac{\alpha}{\eta^{\frac{1}{\beta}} \rho_{\theta}}\right)^{2}\left(\rho_{\theta} \rho_{\theta+2}-\rho_{\theta+1}^{2}\right) \text {. }
$$

5.3. Moment generating function(MGF)

Proposition 3. Let V be a r.v. with pdf given by (1.3). Then the MGF denoted by $M(t)$ of $\mathrm{V}$ is given by

$$
M(t)=\sum_{r=0}^{\infty} \frac{t^{r} \alpha^{r} \rho_{\theta+r}}{r ! \eta^{\frac{r}{\beta}} \rho_{\theta}}
$$

Proof. The MGF is given by

$$
M(t)=E\left(e^{t v}\right) .
$$

By using Taylor's series expansion of the function $e^{t v}$, we obtain

$$
\begin{aligned}
M(t) & =\sum_{r=0}^{\infty} \frac{t^{r}}{r !} \int_{0}^{\infty} v^{r} f_{w}(v) d v \\
& =\sum_{r=0}^{\infty} \frac{t^{r} \alpha^{r} \rho_{\theta+r}}{r ! \eta^{\frac{r}{\beta}} \rho_{\theta}} .
\end{aligned}
$$

This proves the theorem.

5.4. Incomplete moments

Proposition 4. If $\mathrm{V}$ is a r.v. with pdf given by (1.3). Then the $\mathrm{r}^{\text {th }}$ incomplete moment is given by

$$
\mathrm{M}_{\mathrm{r}}(Z)=\frac{\alpha^{r}}{\eta^{\frac{r}{\beta}} \rho_{\theta}} \gamma\left(\frac{\theta+r}{\beta}+1, \eta\left(\frac{Z}{\alpha}\right)^{\beta}\right)
$$

Proof. We have

$$
\mathrm{M}_{\mathrm{r}}(Z)=\int_{0}^{Z} v^{r} f_{w}(v) d v=\frac{\beta \eta^{\frac{\theta}{\beta}+1}}{\alpha^{\beta+\theta} \rho_{\theta}} \int_{0}^{z} v^{\beta+\theta+r-1} \exp \left(-\eta\left(\frac{v}{\alpha}\right)^{\beta}\right) d v .
$$

After the simplification, we get 


$$
\mathrm{M}_{\mathrm{r}}(Z)=\frac{\alpha^{r}}{\eta^{\frac{r}{\beta}} \rho_{\theta}} \gamma\left(\frac{\theta+r}{\beta}+1, \eta\left(\frac{Z}{\alpha}\right)^{\beta}\right)
$$

This completes the proof.

5.5. Standard deviation and coefficient of variation

Standard deviation of WNWP distribution is given by

$$
\sigma=\frac{\alpha}{\eta^{\frac{1}{\beta}} \rho_{\theta}}\left(\rho_{\theta} \rho_{\theta+2}-\rho_{\theta+1}^{2}\right)^{\frac{1}{2}} .
$$

And the coefficient of variation (C.V.) is given as

$$
\mathrm{C} . \mathrm{V} .=\frac{\left(\rho_{\theta} \rho_{\theta+2}-\rho_{\theta+1}^{2}\right)^{\frac{1}{2}}}{\rho_{\theta+1}} .
$$

5.6. Skewness and Kurtosis

\begin{tabular}{|c|c|c|c|c|c|c|c|c|c|c|}
\hline$\alpha$ & $\beta$ & $\eta$ & $\theta$ & Mean & Variance & Mode & STD & C.V & C.S & C.K \\
\hline \multirow{3}{*}{1.5} & \multirow{3}{*}{2.0} & \multirow{3}{*}{1.5} & 1.0 & 1.381977 & 0.340140 & 1.224745 & 0.583216 & 0.4220157 & 0.23589752 & 17.452060 \\
\hline & & & 3.0 & 1.842635 & 0.354694 & 1.732051 & 0.595562 & 0.3232123 & 0.12548755 & 7.267858 \\
\hline & & & 6.0 & 2.374317 & 0.362620 & 2.291288 & 0.602179 & 0.2536224 & 0.07247341 & 0.346280 \\
\hline \multirow{3}{*}{3.5} & \multirow{3}{*}{2.0} & \multirow{3}{*}{1.5} & 1.0 & 3.224612 & 1.851877 & 2.857738 & 1.360837 & 0.4220157 & 0.23589752 & 17.452060 \\
\hline & & & 3.0 & 4.299483 & 1.931115 & 4.041452 & 1.389646 & 0.3232123 & 0.12548755 & 7.267858 \\
\hline & & & 6.0 & 5.540072 & 1.974268 & 5.346338 & 1.405086 & 0.2536224 & 0.07247341 & 0.346280 \\
\hline \multirow{3}{*}{1.5} & \multirow{3}{*}{3.5} & \multirow{3}{*}{1.5} & 1.0 & 1.322362 & 0.131348 & 1.335917 & 0.362419 & 0.2740701 & 0.00013259 & 46.721030 \\
\hline & & & 3.0 & 1.506848 & 0.112910 & 1.520070 & 0.336021 & 0.2229963 & 0.00168424 & 50.404920 \\
\hline & & & 6.0 & 1.710039 & 0.096012 & 1.721392 & 0.309859 & 0.1811998 & 0.00273164 & 22.227070 \\
\hline \multirow{3}{*}{1.5} & \multirow{3}{*}{5.5} & \multirow{3}{*}{1.5} & 1.0 & 1.343069 & 0.064833 & 1.393396 & 0.254624 & 0.1895835 & 0.10110789 & 105.629400 \\
\hline & & & 3.0 & 1.433455 & 0.053631 & 1.474230 & 0.231584 & 0.1615562 & 0.09346717 & 140.050300 \\
\hline & & & 6.0 & 1.535293 & 0.043358 & 1.567235 & 0.208225 & 0.1356259 & 0.07909058 & 126.843900 \\
\hline \multirow{3}{*}{1.5} & \multirow{3}{*}{2.0} & \multirow{3}{*}{4.0} & 1.0 & 0.846284 & 0.127553 & 0.750000 & 0.357145 & 0.4220157 & 0.23589752 & 17.452060 \\
\hline & & & 3.0 & 1.128379 & 0.133010 & 1.060660 & 0.364706 & 0.3232123 & 0.12548755 & 7.267858 \\
\hline & & & 6.0 & 1.453966 & 0.135983 & 1.403122 & 0.368758 & 0.2536224 & 0.07247341 & 0.346280 \\
\hline \multirow{3}{*}{1.5} & \multirow{3}{*}{2.0} & \multirow{3}{*}{7.0} & 1.0 & 0.639730 & 0.072887 & 0.566947 & 0.269976 & 0.4220157 & 0.23589752 & 17.452060 \\
\hline & & & 3.0 & 0.852974 & 0.076006 & 0.801784 & 0.275692 & 0.3232123 & 0.12548755 & 7.267858 \\
\hline & & & 6.0 & 1.099095 & 0.077704 & 1.060660 & 0.278755 & 0.2536224 & 0.07247341 & 0.346280 \\
\hline
\end{tabular}

The coefficient of skewness (C.S.) and kurtosis (C.K.) of WNWPD are given by

$$
\begin{gathered}
\text { C.S. }=\frac{\left(\rho_{\theta}^{2} \rho_{\theta+3}-3 \rho_{\theta} \rho_{\theta+1} \rho_{\theta+2}+2 \rho_{\theta+1}{ }^{3}\right)^{2}}{\left(\rho_{\theta} \rho_{\theta+2}-\rho_{\theta+1}{ }^{2}\right)^{3}} . \\
\text { C.K. }=\frac{\rho_{\theta}^{3} \rho_{\theta+4}-4 \rho_{\theta}{ }^{2} \rho_{\theta+1} \rho_{\theta+3}+6 \rho_{\theta} \rho_{\theta+1}{ }^{2} \rho_{\theta+2}-3 \rho_{\theta+1}{ }^{4}}{\left(\rho_{\theta} \rho_{\theta+2}-\rho_{\theta+1}{ }^{2}\right)^{2}} .
\end{gathered}
$$

Table 1: Statistical properties of WNWP distribution for different values of parameters. 


\section{LORENZ CURVE}

For a continuous random variable $\mathrm{X}$, Lorenz curve is defined as

$$
L(X)=\frac{1}{\mu} \int_{-\infty}^{x} t f(t) d t .
$$

Where $\mu$ is the mean.

Proposition 5. If V follows WNWP distribution with pdf given in (1.3), then its Lorenz curve is given by:

$$
L(V)=\frac{\gamma\left(\frac{\theta+1}{\beta}+1, \eta\left(\frac{v}{\alpha}\right)^{\beta}\right)}{\Gamma\left(\frac{\theta+1}{\beta}+1\right)} .
$$

Proof. Using eq. (1.3), we have

$$
L(V)=\frac{\beta \eta^{\frac{\theta}{\beta}+1}}{\mu \alpha^{\beta+\theta} \Gamma\left(\frac{\theta}{\beta}+1\right)} \int_{0}^{\infty} v^{\beta+\theta-1} \exp \left(-\eta\left(\frac{v}{\alpha}\right)^{\beta}\right) d v .
$$

On solving the above integral and substituting the value of $\mu$, we get

$$
L(V)=\frac{\gamma\left(\frac{\theta+1}{\beta}+1, \eta\left(\frac{v}{\alpha}\right)^{\beta}\right)}{\Gamma\left(\frac{\theta+1}{\beta}+1\right)} .
$$

Hence proved.

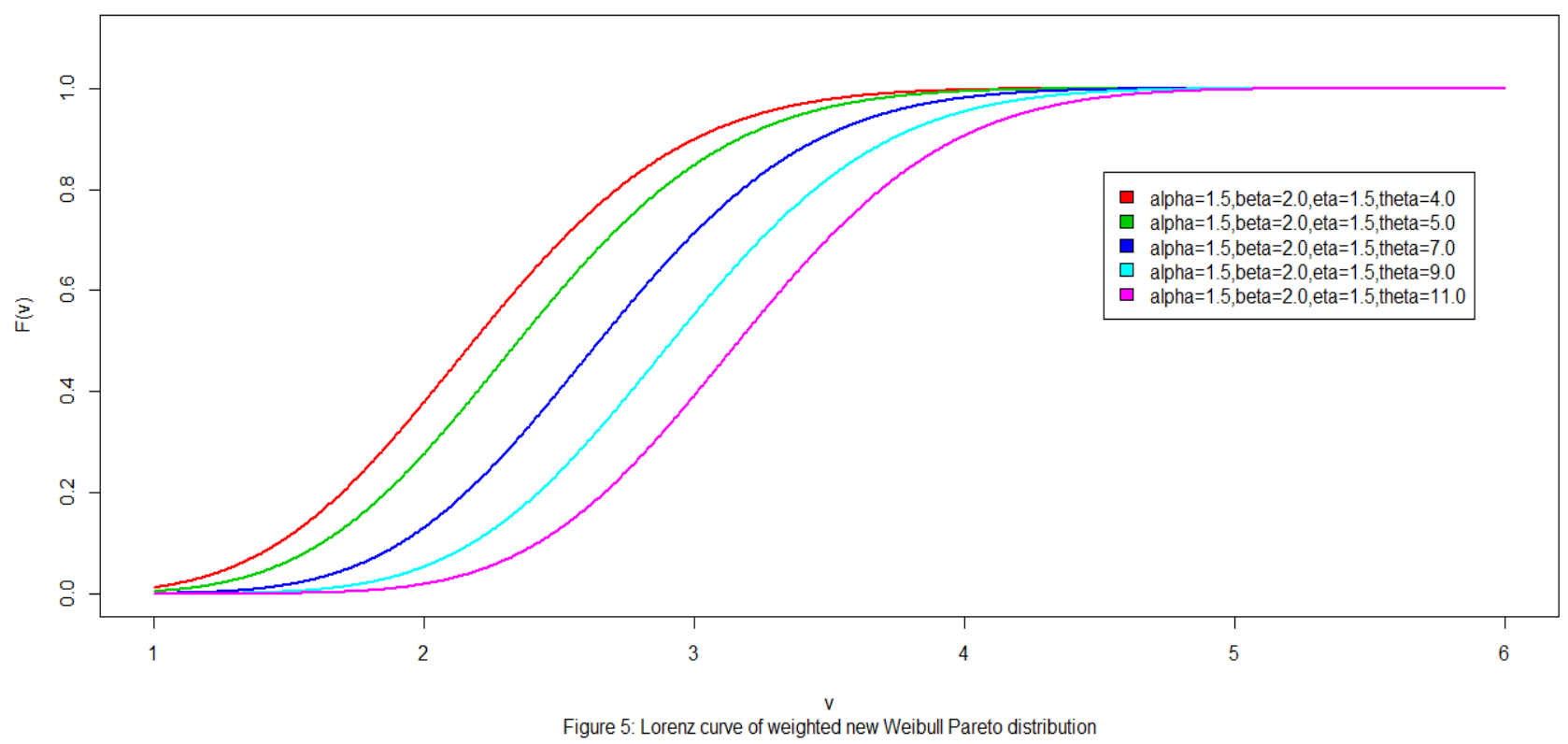

\section{SHANNON'S ENTROPY}

Shannon's entropy is the most popular measure of entropy and is defined for a random variable $\mathrm{V}$ having pdf $f(v)$ as $H(f)=E(-\log (f(v)))$. 
Proposition 6. For a r.v. V with pdf given in (1.3), the Shannon's entropy is given by

$$
H\left(f_{w}(v)\right)=\log \left(\frac{\alpha \Gamma\left(\frac{\theta}{\beta}+1\right)}{\beta}\right)+\frac{1}{\beta}(\theta-\log \eta)+\left(\frac{\beta+\theta-1}{\beta}\right) \varphi\left(\frac{\theta}{\beta}+1\right)+1 .
$$

Where $\varphi\left(\frac{\theta}{\beta}+1\right)=\frac{\Gamma^{\prime}\left(\frac{\theta}{\beta}+1\right)}{\Gamma\left(\frac{\theta}{\beta}+1\right)}$ is a digamma function.

Proof. Using equation (7.1), we have

$$
\begin{aligned}
H\left(f_{w}(v)\right) & =E\left(-\log \left(f_{w}(v)\right)\right. \\
& =-\log \left(\frac{\beta \eta^{\frac{\theta}{\beta}+1}}{\alpha^{\beta+\theta} \Gamma\left(\frac{\theta}{\beta}+1\right)}\right)-(\beta+\theta-1) E(\log (v))+\frac{\eta}{\alpha^{\beta}} E\left(v^{\beta}\right) .
\end{aligned}
$$

Now, $\quad E(\log (v))=\int_{0}^{\infty} \log (v) f_{w}(v) d v$.

By substituting the value of eq. (1.3) in eq. (7.3), we get

$$
E(\log (v))=\log (\alpha)-\frac{1}{\beta} \log (\eta)+\frac{1}{\beta} \varphi\left(\frac{\theta}{\beta}+1\right) .
$$

Similarly, $E\left(v^{\beta}\right)=\frac{\alpha^{\beta}}{\beta \theta}(\theta+\beta)$.

On substituting the value of eq. (7.4) and eq. (7.5) in eq. (7.2), we get

This proves the theorem.

$$
H\left(f_{w}(v)\right)=\log \left(\frac{\alpha \Gamma\left(\frac{\theta}{\beta}+1\right)}{\beta}\right)+\frac{1}{\beta}(\theta-\log \eta)+\left(\frac{\beta+\theta-1}{\beta}\right) \varphi\left(\frac{\theta}{\beta}+1\right)+1 .
$$

\section{ENTROPY ESTIMATION OF WNWP DISTRIBUTION}

Suppose that we have a statistical model having likelihood function $\mathrm{L}$ and $\mathrm{N}$ be the number of parameters. Then Akaike information criteria (AIC) and Bayesian information criteria (BIC) of the model is given by

$$
\mathrm{AIC}=2 N-2 \log (L) \text {. }
$$

$$
\mathrm{BIC}=N \log (n)-2 \log (L) \text {. }
$$

From eq. (1.3), we have

$$
\log (L)=n \log \left(\frac{\beta \eta^{\frac{\theta}{\beta}+1}}{\alpha^{\beta+\theta} \Gamma\left(\frac{\theta}{\beta}+1\right)}\right)+(\beta+\theta-1) \sum_{i=1}^{n} \log \left(v_{i}\right)-\frac{\eta}{\alpha^{\beta}} \sum_{i}^{n} v_{i}{ }^{\beta} .
$$




$$
\Rightarrow \quad \frac{-\log (L)}{n}=-\log \left(\frac{\beta \eta^{\frac{\theta}{\beta}+1}}{\alpha^{\beta+\theta} \Gamma\left(\frac{\theta}{\beta}+1\right)}\right)-(\beta+\theta-1) E(\log (v))+\frac{\eta}{\alpha^{\beta}} E\left(v^{\beta}\right) .
$$

On comparing eq. (7.2) and (8.3), we get

$$
H\left(f_{w}(v)\right)=\frac{-\log (L)}{n} .
$$

Thus from eq. (8.1) and eq. (8.2), we get

$$
\begin{aligned}
& \mathrm{AIC}=2 N+2 n H\left(f_{w}(v)\right) . \\
& \mathrm{BIC}=N \log (n)+2 n H\left(f_{w}(v)\right) .
\end{aligned}
$$

\section{CHARACTERIZATION OF WNWP DISTRIBUTION}

Proposition 7. If $v_{1}, v_{2}, \ldots, v_{n}$ are i.i.d. random samples drawn from (1.3), then

$$
\lim _{n \rightarrow \infty} E\left(\frac{s_{n}{ }^{2}}{\bar{v}^{2}}\right)=\lim _{n \rightarrow \infty} E\left(\frac{s_{n}}{\bar{v}}\right)^{2} \rightarrow\left(\frac{\sigma}{\mu}\right)^{2} .
$$

Proof. We have

$$
\begin{aligned}
E\left(\bar{v}_{n}\right) & =\frac{\sigma^{2}}{n}+\mu^{2} \\
& =\frac{\alpha^{2}\left(\rho_{\theta} \rho_{\theta+2}-\rho_{\theta+1}{ }^{2}+n \rho_{\theta+1}{ }^{2}\right)}{n \eta^{\frac{2}{\beta}} \rho_{\theta}{ }^{2}} .
\end{aligned}
$$

Now, $E\left(\frac{s_{n}{ }^{2}}{\bar{v}^{2}}\right)=\frac{E\left(s_{n}{ }^{2}\right)}{E\left(\bar{v}^{2}\right)}$

$$
=\frac{\frac{\alpha^{2}\left(\rho_{\theta} \rho_{\theta+2}-\rho_{\theta+1}^{2}\right)}{\eta^{\frac{2}{\beta}} \rho_{\theta}{ }^{2}}}{\frac{\alpha^{2}\left(\rho_{\theta} \rho_{\theta+2}-\rho_{\theta+1}{ }^{2}+n \rho_{\theta+1}{ }^{2}\right)}{n \eta^{\frac{2}{\beta}} \rho_{\theta}{ }^{2}}} .
$$

Therefore, $\lim _{n \rightarrow \infty} E\left(\frac{s_{n}{ }^{2}}{\bar{v}^{2}}\right)=\left(\frac{\sigma}{\mu}\right)^{2}$

\section{REAL LIFE ILLUSTRATION}

In this section we have given the real data application of WNWP distribution. We have fitted the WNWP distribution for two different real life data sets. The data set first comprised of 72 exceedances of flood peaks $\left(\mathrm{m}^{3} / \mathrm{s}\right)$ of the Wheaton river near car cross in Yukon territory, Canada for the calender 1958-1984. The data set second represents the survival times (in days) of guinea pigs injected with different doses of tubercle bacilli. 
Table 2: Descriptive Statistics for data set 1.

\begin{tabular}{cccccccc}
\hline Min. & 1st Qu. & Median & Mean & 3rd Qu. & Max. & Skewness & Kurtosis \\
\hline 0.10 & 1.85 & 9.50 & 12.09 & 20.12 & 64.00 & 1.4657 & 5.8268 \\
\hline
\end{tabular}

Table 3: Descriptive Statistics for data set 2.

\begin{tabular}{cccccccc}
\hline Min. & 1st Qu. & Median & Mean & 3rd Qu. & Max. & Skewness & Kurtosis \\
\hline 12.00 & 54.75 & 70.00 & 99.82 & 112.80 & 376.00 & 1.7962 & 5.6144 \\
\hline
\end{tabular}

Table 4. Maximum likelihood estimates, standard error in parentheses and statistics for model selection using data set 1.

\begin{tabular}{|c|c|c|c|c|c|c|c|c|}
\hline \multirow[b]{2}{*}{ Model } & \multicolumn{4}{|c|}{ Estimate, standard error in parentheses } & \multirow[b]{2}{*}{$-2 \log 1$} & \multirow[b]{2}{*}{$\begin{array}{c}\text { Shannon's } \\
\text { entropy }\end{array}$} & \multirow[b]{2}{*}{$\mathrm{AIC}$} & \multirow[b]{2}{*}{$\mathrm{BIC}$} \\
\hline & $\hat{\alpha}$ & $\hat{\beta}$ & $\hat{\eta}$ & $\hat{\theta}$ & & & & \\
\hline WNWPD & $\begin{array}{l}0.004497 \\
(0.00119)\end{array}$ & $\begin{array}{l}0.322636 \\
(0.05239)\end{array}$ & $\begin{array}{l}1.063163 \\
(0.82534)\end{array}$ & $\begin{array}{l}3.522454 \\
(1.08054)\end{array}$ & 536.0425 & 3.7225 & 544.0425 & 553.1492 \\
\hline LNWPD & $\begin{array}{l}1.253596 \\
(0.39025) \\
\end{array}$ & $\begin{array}{l}2.206362 \\
(0.19543) \\
\end{array}$ & $\begin{array}{l}0.004041 \\
(0.00106)\end{array}$ & - & 777.1507 & 5.3969 & 783.1507 & 789.9806 \\
\hline NWPD & $\begin{array}{l}0.787792 \\
(0.19685) \\
\end{array}$ & $\begin{array}{l}0.050331 \\
(0.01439) \\
\end{array}$ & $\begin{array}{l}8.435934 \\
(2.75611) \\
\end{array}$ & - & 1709.05 & 11.8684 & 1715.0501 & 1721.8799 \\
\hline
\end{tabular}

Table 5. Maximum likelihood estimates, standard error in parentheses and statistics for model selection using data set 2.

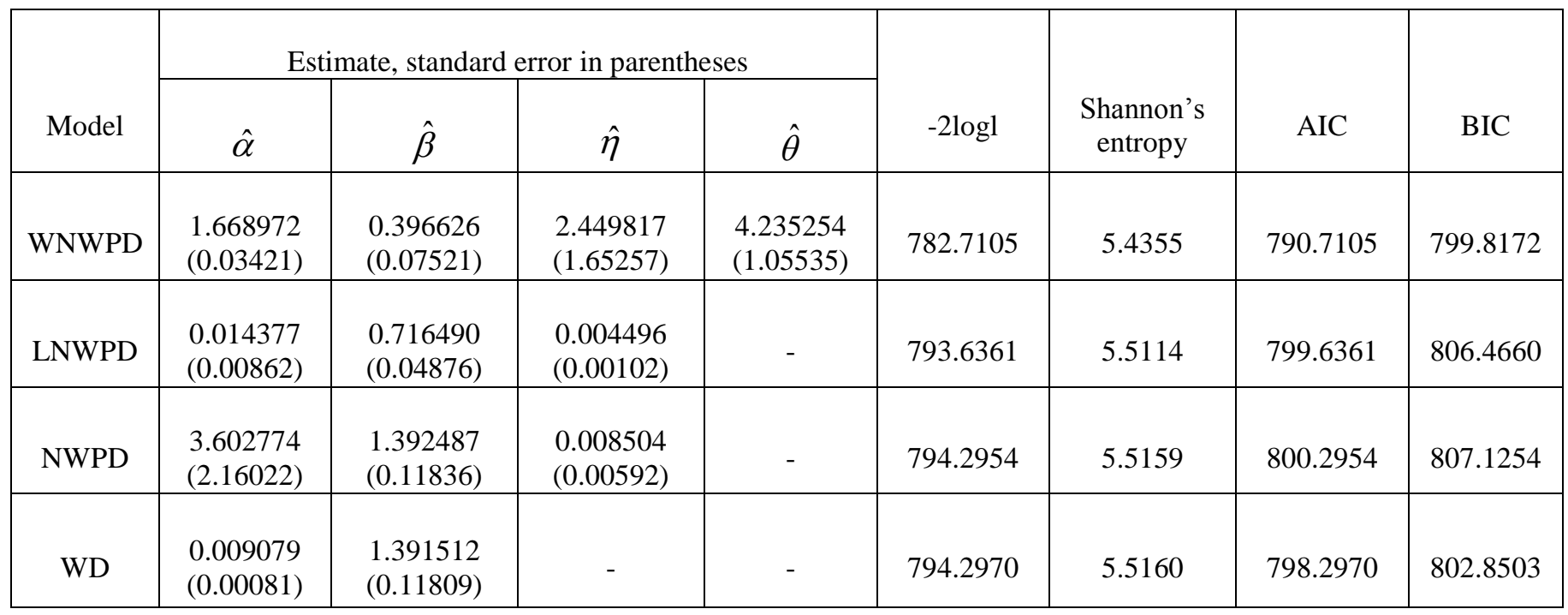




\section{CONCLUSION}

This paper deals with the weighted new Weibull Pareto (WNWP) distribution and studies its different statistical properties include reliability analysis, mode, moments, moment generating function, incomplete moments,standared deviation, coeffecient of variation, skewness, kurtosis, Lorenz curve, Shannon's entropy. Graphs were plotted using R-software. The superiority of the new model over some other models viz LNWPD, NWPD and WD were checked. An application to real life data sets shows that the fit of WNWP distribution is superior to the fits using LNWPD, NWPD and WD.

\section{REFERENCES}

[1] R.A. Fisher, "The effects of methods of ascertainment upon the estimation of frequencies", The Annals of Eugenics, vol.6, pp. 13-25, 1934.

[2] C.R. Rao, "On discrete distributions arising out of methods of ascertainment, in classical and contagious discrete distributions", Pergamon press and statistical publishing society, Calcutta, pp. 320-332, 1965.

[3] K. Jain, N. Singla, and D.R. Gupta, "A weighted version of gamma distribution", Discussiones mathematicae probability and statistics, vol.34, pp. 89-111, 2014.

[4] M.M.E. Monsef and S.A.E. Ghoneim, “The weighted Kumaraswamy distribution”, International information institute, vol. 18, pp. 32893300, 2015.

[5] kawsar Fatima and S.P. Ahmad, "Weighted inverse Rayleigh distribution”, International journal of statistics and systems, vol. 12, pp. 119137, 2017.

[6] Sofi Mudasir and S.P. Ahmad, "Weighted Nakagami distribution with applications using R-Software", AGU International journal of management studies \& research, vol, 5, pp. 941-952, 2017.

[7] Sofi Mudasir and S.P. Ahmad, "Parameter estimation of weighted Erlang distribution using R-software", Mathematical theory and modeling, vol. 7, pp. 1-21, 2017.

[8] Uzma Jan, Kawsar Fatima and S.P. Ahmad, "on weighted Ailamujia distribution and its applications to life time data", Journal of statistics applications and probability, vol. 6, pp. 619-633, 2017.

[9] Suleman Nasiru and Albert Luguterah' "The New Weibull-Pareto distribution", Pakistan journal of statistics and operation research, vol, 11, pp. 103-114, 2015. 